\title{
Tumour-infiltrating lymphocytes in oropharyngeal cancer: a validation study according to the criteria of the International Immuno-Oncology Biomarker Working Group
}

\author{
Alhadi Almangush (DD ${ }^{1,2,3,4}{ }^{凶}$, Lauri Jouhi ${ }^{5}$, Timo Atula ${ }^{5}$, Caj Haglund ${ }^{6,7}$, Antti A. Mäkitie (iD ${ }^{2,5,8}$, Jaana Hagström ${ }^{1,6,9}$ and IImo Leivo ${ }^{10,11}$
}

(c) The Author(s) 2022

BACKGROUND: The evaluation of immune response can aid in prediction of cancer behaviour. Here, we assessed the prognostic significance of tumour-infiltrating lymphocytes (TILs) in oropharyngeal squamous cell carcinoma (OPSCC).

METHODS: A total of 182 patients treated for OPSCC were included in this study. Assessment of TILs was conducted on tumour sections stained with standard haematoxylin and eosin (HE) staining. We used the scoring criteria proposed by the International Immuno-Oncology Biomarker Working Group.

RESULTS: The multivariable analysis showed that TILs associated with disease-specific survival with a hazard ratio (HR) of 2.13 (95\% CI 1.14-3.96; $P=0.017)$. Similarly, TILs associated significantly with overall survival with HR of $1.87(95 \% \mathrm{Cl} 1.11-3.13 ; P=0.018)$. In a subanalysis of HPV-positive and HPV-negative cases separately, TILs showed a significant prognostic value in both groups $(P<0.05)$.

CONCLUSION: The evaluation of TILs as proposed by the International Immuno-Oncology Biomarker Working Group is a simple and promising method in prediction of survival of OPSCC. It is easily applicable and after further validation can be implemented in the routine pathological report as a basic immune parameter.

British Journal of Cancer (2022) 126:1589-1594; https://doi.org/10.1038/s41416-022-01708-7

\section{INTRODUCTION}

Oropharyngeal squamous cell carcinoma (OPSCC) is one of the most common cancers of head and neck region. OPSCC is often associated with human papillomavirus (HPV) infection, but it can also be caused by other risk factors such as tobacco and alcohol abuse. Of note, the incidence of HPV-related OPSCC is increasing rapidly in many countries worldwide [1-5]. Fortunately, patient survival in HPV-related OPSCC is better compared with virusnegative OPSCC, but the estimation of the clinical behaviour of OPSCC is sometimes challenging. Especially with classification of OPSCC as either HPV-positive or HPV-negative there are few additional prognostic factors that can be considered in risk assessment. Adverse prognostic factors include old age, advanced stage and smoking [6]. In daily practice, however, prognostication schemes currently available for OPSCC do not include assessment of the immune status.

Tumour immune microenvironment has been linked strongly with cancer behaviour [7]. For selection of patients regarding treatment strategies, tumour-infiltrating lymphocytes (TILs) have been proposed as biomarkers in many tumour types including those of the head and neck [8]. The International ImmunoOncology Biomarker Working Group has proposed a method for standardised assessment of TILs in haematoxylin and eosin (HE) stained slides $[9,10]$ with a good interobserver agreement in a number of studies [11-14]. This method can be considered in the daily practice of the pathologist evaluating TILs [15]. In the present study, we wondered if the local immune cell infiltration in OPSCC could associate with tumour behaviour, and whether it can be measured in routine HE-stained sections. Therefore, we studied a large cohort of OPSCCs including both HPV-positive and HPVnegative tumours, with a sub-analysis to assess the universal use of this prognostic marker.

\section{METHODS}

Our cohort included all patients treated for oropharyngeal cancer at the Helsinki University Hospital (Helsinki, Finland) during the 10-year period from January 2000 to December 2009. We excluded patients who had

\footnotetext{
${ }^{1}$ Department of Pathology, University of Helsinki, P.O. Box 21, Fl-00014 Helsinki, Finland. ${ }^{2}$ Research Program in Systems Oncology, Faculty of Medicine, University of Helsinki, Helsinki, Finland. ${ }^{3}$ Department of Pathology, University of Turku, Turku, Finland. ${ }^{4}$ Faculty of Dentistry, Misurata University, Misurata, Libya. ${ }^{5}$ Department of Otorhinolaryngology Head and Neck Surgery, University of Helsinki and Helsinki University Hospital, P.O. Box 263, Fl-00029 Helsinki, Finland. ${ }^{6}$ Research Programs Unit, Translational Cancer Medicine, University of Helsinki, P.O. Box 63, 00014 Helsinki, Finland. ${ }^{7}$ Department of Surgery, University of Helsinki and Helsinki University Hospital, Helsinki, Finland. ${ }^{8}$ Division of Ear, Nose and Throat Diseases, Department of Clinical Sciences, Intervention and Technology, Karolinska Institutet and Karolinska University Hospital, Stockholm, Sweden. ${ }^{9}$ Department of Oral Pathology and Radiology, University of Turku, Turku University Hospital, Turku, Finland. ${ }^{10}$ Institute of Biomedicine, Pathology, University of Turku, Kiinamyllynkatu 10 D 5035 , 20520 Turku, Finland. ${ }^{11}$ Turku University Central Hospital, 20521 Turku, Finland. ${ }^{\circledR}$ email: alhadi.almangush@helsinki.fi
} 
received palliative treatment $(n=44)$, and patients with concurrent head and neck cancers $(n=5)$, with earlier treatments for head and neck cancer $(n=11)$, with histologies other than squamous cell carcinoma $(n=18)$, and cases where tumour tissue was not available $(n=71)$. Tissue samples were collected before radiotherapy or chemoradiotherapy in all but two cases, where post treatment specimens only were available for evaluation. The pretreatment samples included both diagnostic pretreatment biopsies and resected tissues from primary surgery. The patients in this retrospective study were treated between 2000 and 2009. Immunotherapies were not used at that time, so none of the patients received immunotherapy.

A total of 182 cases of OPSCC were included in our analysis of TILs. We used Ventana Inform HPV in situ hybridisation assay to determine HPV status. This study was conducted in compliance with the Declaration of Helsinki and approved by the Research Ethics Committee of the Helsinki University Hospital.

For the evaluation of TILs, we followed the method introduced by the International Immuno-Oncology Biomarkers Working Group for standardisation of the assessment of TILs in routine HE-stained sections [9, 10]. In brief, the whole slide was scanned at low magnification with $\times 5$ or $\times 10$ objective lens, followed by a higher magnification with $\times 20$ objective lens. Stromal TILs were defined as the percentage of stromal area occupied by infiltrating lymphocytes. The average number of TILs was assessed in multiple stromal areas. Mononuclear immune cells were scored, while polymorphonuclear leucocytes were excluded. In addition, areas of necrosis were excluded. Furthermore, TILs in stromal areas not adjacent to the tumour were excluded. Assessment of TILs was carried out in areas of tumour growth in connective tissues (Fig. 1), while the lymphatic tissue of tonsils was excluded.

All available diagnostic slides stained with $\mathrm{H}$-E were evaluated. TILs were assessed in percentages as a continuous score $(5 \%, 10 \%, 20 \%, 30 \%$... etc.). To identify the optimal cutoff point of TIL score with regard to survival, we tested different cutoffs $(5 \%, 10 \%, 20 \%, 30 \%$.... etc.) dividing tumours with low TILs and high TILs. Two observers (AA, IL) arranged a training session for the assessment of TILs guided by an experienced head and neck pathologist (IL), and a subsequent reviewing session. Both observers were fully blinded to the clinicopathologic characteristics and the outcome of the cases.

\section{Statistical analysis}

We used IBM SPSS Statistics (version 25) for all statistical analyses. The Kappa Coefficient test was used to evaluate interobserver variance. A twosided $P$ value of $<0.05$ was considered statistically significant. The relationship between TILs and clinicopathologic characteristics was analysed using cross-tabulation and evaluated by Chi-Square test. We used the Kaplan-Meier estimate and log-rank test for survival analyses. Cox regression was used in univariable and multivariable setting. Multivariable model was used as a method to control confounding factors. Diseasespecific survival was measured from the completion of primary treatment to death from disease or last follow-up, while overall survival was measured from the completion of primary treatment to any death or last follow-up. The prognostic value of TILs was analysed separately, and also in combination with $\mathrm{T}$ classification as follows:
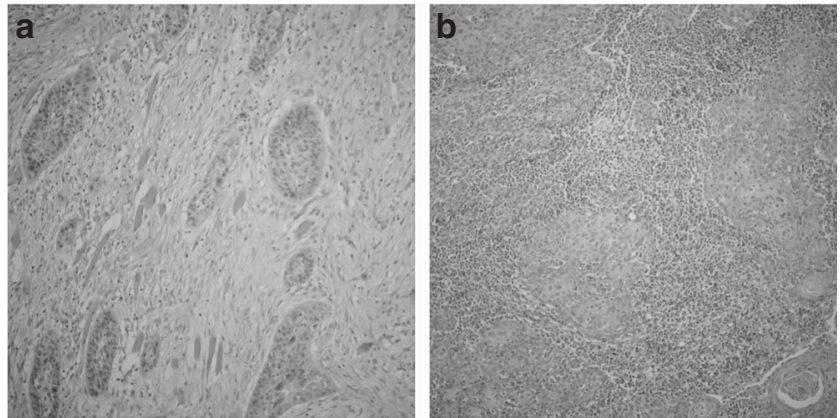

Fig. 1 Examples of expression of tumour-infiltrating lymphocytes (TILs) in haematoxylin and eosin-stained sections (magnification $\times 100$ ) of oropharyngeal squamous cell carcinoma (OPSCC). a Scarce expression of TILs in OPSCC where very few immune cells were presented in the stroma. b Predominant TILs infiltrate in OPSCC where almost the whole stroma is occupied by TILs.
T1-TILs ${ }^{\text {High }}$ includes tumours as described in AJCC 8 (i.e. $\leq 2 \mathrm{~cm}$ in greatest dimension) and TILs are more than or equal to $60 \%$.

T2-TILs ${ }^{\text {Moderate }}$ includes tumours as described in AJCC 8 (i.e. $>2 \mathrm{~cm}$ in greatest dimension but not larger than $4 \mathrm{~cm}$ ) and with TILs ranging from $>20 \%$ to $<60 \%$.

T3-TILs ${ }^{\text {Low }}$ includes tumours as described in AJCC 8 (i.e. $>4 \mathrm{~cm}$ in greatest dimension or extension to lingual surface of epiglottis) and with TILs less or equal to $20 \%$.

No change in T4 as the tumours in this class severely extend into surrounding tissues.

\section{RESULTS}

The clinicopathologic information of the patients and their relationship with TILs are summarised in Table 1. The cohort included 140 (76.9\%) men and 42 (23.1\%) women. The median follow-up time was 4.48 years (range $3.51-5.00$ years). We assessed stromal TILs because the stroma was the predominant location of TILs in our OPSCC tumours. Infiltration of intra-tumoural TILs was very limited and thus not suitable for a prognostic marker. In the stroma, the expression of TILs ranged from 1 to $90 \%$. No predefined cutoff points were available, but we found $20 \%$ as an optimal cutoff point regarding risk stratification of OPSCC. A low infiltration of TILs (i.e. $<20 \%$ ) was found in $49(26.9 \%)$ tumours, while a high infiltration (i.e. TILs $\geq 20 \%$ ) presented in 133 (73.1\%) tumours. A substantial agreement (Kappa value $=0.78$ ) was found between the two scoring observers.

Table 1. Association between tumour-infiltrating lymphocytes (TILs) and clinicopathologic characteristics of 182 cases treated for oropharyngeal squamous cell carcinoma.

\begin{tabular}{|c|c|c|c|c|}
\hline \multirow[t]{2}{*}{ Variable } & Total & $\begin{array}{l}\text { Low TILs } \\
(<20 \%)\end{array}$ & $\begin{array}{l}\text { High TILs } \\
(\geq 20 \%)\end{array}$ & $\begin{array}{l}P \text { value of chi- } \\
\text { square test }\end{array}$ \\
\hline & $\begin{array}{l}\text { Total, } \\
N=182\end{array}$ & $\begin{array}{l}\text { Number } \\
(\%) 49 \\
(26.9 \%)\end{array}$ & $\begin{array}{l}\text { Number (\%) } \\
133(73.1 \%)\end{array}$ & \\
\hline Gender & & & & 0.236 \\
\hline Male & 140 & $41(29.3 \%)$ & 99 (70.7\%) & \\
\hline Female & 42 & 8 (19\%) & 34 (81\%) & \\
\hline Smoking & & & & 0.538 \\
\hline Never & 20 & $6(30 \%)$ & $14(70 \%)$ & \\
\hline Former & 46 & $10(21.7 \%)$ & $36(78.3 \%)$ & \\
\hline Currently & 85 & $26(30.6 \%)$ & 59 (69.4\%) & \\
\hline T classification & & & & 0.010 \\
\hline $\mathrm{T} 1$ & 35 & $4(11.4 \%)$ & 31 (88.6\%) & \\
\hline $\mathrm{T} 2$ & 68 & $15(22.1 \%)$ & 53 (77.9\%) & \\
\hline T3 & 40 & $13(32.5 \%)$ & 27 (67.5\%) & \\
\hline $\mathrm{T} 4$ & 39 & 17 (43.6\%) & $22(56.4 \%)$ & \\
\hline $\mathrm{N}$ classification & & & & 0.473 \\
\hline No-1 & 57 & $13(22.8 \%)$ & 44 (77.2\%) & \\
\hline N2-3 & 125 & $36(28.8 \%)$ & 89 (71.2\%) & \\
\hline Stage & & & & 0.644 \\
\hline Early (I-II) & 27 & $6(22.2 \%)$ & $21(77.8 \%)$ & \\
\hline $\begin{array}{l}\text { Advanced } \\
\text { (III-IV) }\end{array}$ & 155 & $43(27.7 \%)$ & 112 (72.3\%) & \\
\hline Grade & & & & 0.34 \\
\hline 1 & 15 & $3(20 \%)$ & $12(80 \%)$ & \\
\hline II & 70 & 23 (32.9\%) & 47 (67.1\%) & \\
\hline III & 97 & $23(23.7 \%)$ & 74 (76.3\%) & \\
\hline HPV status & & & & 0.616 \\
\hline Positive & 91 & $23(25.3 \%)$ & $68(74.7 \%)$ & \\
\hline Negative & 91 & $26(28.6 \%)$ & 65 (71.4\%) & \\
\hline Treatment & & & & 0.078 \\
\hline$S x \pm(C) R T$ & 120 & 27 (22.5\%) & 93 (77.5\%) & \\
\hline (C) $R T \pm S x$ & 62 & 22 (35.5\%) & 40 (64.5\%) & \\
\hline
\end{tabular}

Statistically significant $P$ value are in bold

Sx Surgery; CRT chemoradiotherapy, $R T$ radiotherapy. 

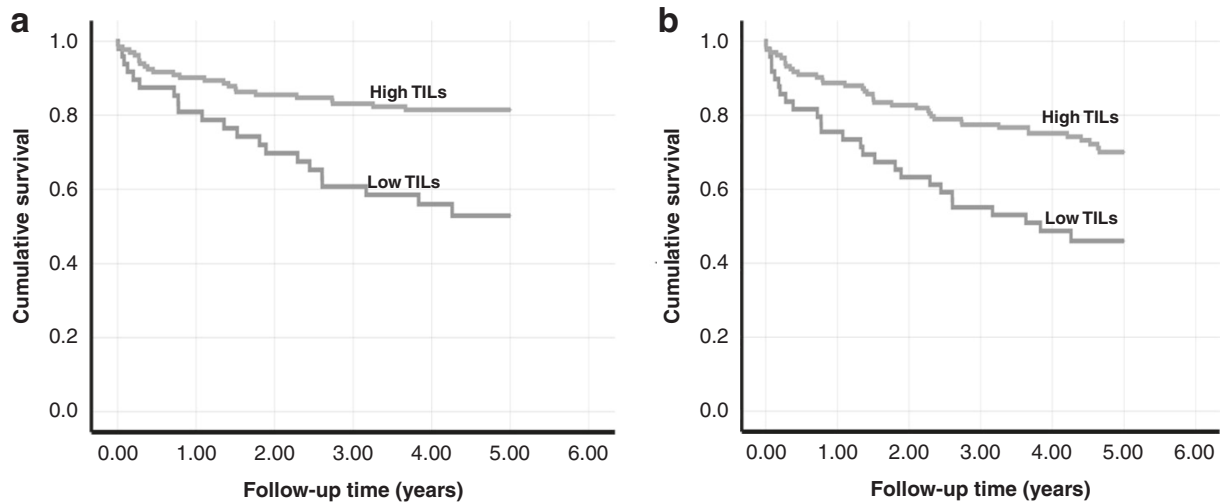

Fig. 2 Kaplan-Meier survival curves showing comparison of cases as classified into a low-risk group or a high-risk group based on the expression of tumour-infiltrating lymphocytes (TILs). a Disease-specific survival $(P<0.001)$. b Overall survival $(P=0.001)$.

There was a significant association between T-classification and TILS with smaller tumours associating with a higher infiltration of TILs $(P=0.01)$. However, no significant association was noted between TILs and the gender of patients, N-classification, overall stage (I-IV), histological grade, HPV-status, smoking habits, or the treatment regimen given to the patient $(P>0.05)$.

The univariable analysis showed a significant association between tumours with low TILs, poor disease-specific survival (HR 2.84, 95\%Cl 1.58-5.11; $P<0.001)$ and worse overall survival (HR 2.27, 95\%Cl 1.38-3.75; $P=0.001$ ) of the patients. In multivariable models a similar association was observed for both disease-specific survival (HR 2.13,95\%Cl 1.14-3.96; $P=0.017)$ and overall survival (HR 1.87, 95\%Cl 1.11-3.13; $P=0.018$ ). Further, Kaplan-Meier curves showed a poor survival for cases with low TILs as shown in Fig. 2 for both disease-specific survival $(P<0.001)$ and overall survival $(P=0.001)$. Interestingly, a significant association of low TILs with poor survival was also seen in both survival analyses $(P<0.05)$ when the cohort was divided into HPV-positive and HPV-negative cases.

Among all clinicopathologic characteristics included in the analyses (Table 2), HPV-status is the only variable that showed a significant association in both disease-specific survival (HR 2.98, 95\%Cl 1.58-5.64; $P=0.001)$ and overall survival $(2.41$, $95 \% \mathrm{Cl} 1.44-4.05 ; P=0.001)$ after all parameters were included in the model. The prognostic value of the other parameters is summarised in Table 2.

When the TIL score was combined with T classification, a total of 25 cases were up-staged from T1-TILs to T2-TILs, and 29 cases were up-staged from T2-TILs to T3-TILs. On the other hand, 16 cases were down-staged from T3-TILs to T2-TILs, and 10 cases from T2-TILs to T1-TILs. Interestingly, a gradual increase in the risk was reported in the analysis of disease-specific survival from T1TILs to T2-TILs (HR 1.33, 95\%Cl 0.38-4.68), T3-TILs (HR 2.23, 95\%Cl $0.65-7.64)$ and T4 (HR 3.04, 95\%Cl 0.87-10.68). Similarly, a gradually increased risk was noted in the analysis of overall survival for T2-TILs (HR 1.52, 95\%Cl 0.52-4.45), T3-TILs (HR 2.06, 95\% $\mathrm{Cl} 0.71-6.04)$ and T4 (HR 3.63, 95\%Cl 1.24-10.64).

\section{DISCUSSION}

The significance of TILs in predicting cancer outcome has been reported in many studies $[8,16,17]$. A proposal for a standardised method to evaluate TILs in solid tumours using HE-stained sections was introduced recently $[9,10]$ and a good reproducibility in different cancer types has been reported [11-14]. In the present study, we report the use of this method in assessing TILs in oropharyngeal cancer.

Tumour microenvironment consists of different cell typesincluding immune cells-that influence cancer progression [18].
Immune response to cancer and the assessment of such a response has been a topic for active research in recent years. Specifically, the evaluation of infiltrating lymphocytes can reveal the status of the pre-existing immunogenicity of the tumour [19]. Similar to previous studies [12,19-21], we found stromal TILs to be clinically relevant, while intra-tumoural TILs were less important to patient survival. This can be explained by the fact that the immune microenvironment is a major player in tumourhost interactions [22]. The limited prognostic significance of intra-tumoural TILs may be due to the fact that they constitute only a small proportion of total tumour-related TILs. One also needs to appreciate the relative difficulty and inaccuracy in assessment of TILs embedded in intra-tumoural sites in HEslides $[23,24]$.

TILs consist of different immune cells (with predominance of T lymphocytes) that have left the blood stream and infiltrated into the tumour tissue and play a major role in the immune response to cancer [25]. Abundance of TILs indicates that antitumour immune response is strong and therefore may contribute to favourable survival [26]. The significance of TILs as a reliable prognostic marker in many tumour types has increased in recent years. The method of overall assessment of TILs in HE-stained sections has shown to be of reliable prognostic value in breast cancer [19], colorectal cancer [14], gastric cancer [27], lung cancer [28], and different subsites of head and neck cancer [8]. In particular, three studies have reported the significance of assessing TILs in HPV-associated OPSCC [29-31] and their findings were in line with our results. However, the method of assessing TILs is not yet standardised for OPSCC. In the present study, we followed the method of the International ImmunoOncology Biomarker Working Group [9, 10] and found that scoring TILs in HE-stained sections can classify OPSCC tumours into low-risk and high-risk groups. This score is significant for both HPV-positive and HPV-negative OPSCC, demonstrating that TILs can be used as a universal prognostic tool for OPSCC. In our analysis, a high infiltration of tumours by TILs was associated with a better survival in OPSCC, and similar results have been reported in other subsites of head and neck cancer [8], and other cancers as well $[14,19,32]$. The cutoff point of $20 \%$ that we identified in this study is similar to those in other studies using the same scoring criteria $[12,33]$.

To allow for standardised evaluation of TILs in OPSCC, it is important to consider the method that we used in this study, and that has been published in a practical guide for pathologists by the International Immuno-Oncology Biomarkers Working Group with specific recommendations for various locations of cancer including the head and neck [10]. Following this standardised method will accumulate methodologically homogenous data from different populations for future robust meta-analyses. This could 
Table 2. Univariable and multivariable analyses of 182 cases treated for oropharyngeal squamous cell carcinoma.

\begin{tabular}{|c|c|c|}
\hline \multirow[t]{2}{*}{ Factor } & \multicolumn{2}{|l|}{ Univariable analysis } \\
\hline & $\begin{array}{l}\text { Disease-specific survival } \\
\text { HR }(95 \% \mathrm{Cl}) ; P \text { value }\end{array}$ & $\begin{array}{l}\text { Overall survival } \\
\text { HR }(95 \% \mathrm{Cl}) ; P \text { value }\end{array}$ \\
\hline \multicolumn{3}{|l|}{ Gender } \\
\hline Male & 1 & 1 \\
\hline Female & $2.19(0.99-4.88) ; P=0.054$ & $1.50(0.85-2.64) ; P=0.16$ \\
\hline \multicolumn{3}{|l|}{ Smoking } \\
\hline Never & 1 & 1 \\
\hline Former & $1.66(0.46-6.05) ; P=0.44$ & $1.24(0.48-3.21) ; P=0.65$ \\
\hline Currently & $3.29(1.01-10.7) ; P=0.048$ & $2.36(1.01-5.53) ; P=0.048$ \\
\hline \multicolumn{3}{|l|}{ T classification } \\
\hline $\mathrm{T} 1$ & 1 & 1 \\
\hline $\mathrm{T} 2$ & $1.97(0.74-5.27) ; P=0.18$ & $1.92(0.84-4.43) ; P=0.12$ \\
\hline T3 & $1.79(0.62-5.26) ; P=0.28$ & $2.44(1.03-5.81) ; P=0.044$ \\
\hline T4 & $3.62(1.31-9.96) ; P=0.013$ & $4.18(1.79-9.76) ; P=0.001$ \\
\hline \multicolumn{3}{|l|}{$\mathrm{N}$ classification } \\
\hline No-N1 & 1 & 1 \\
\hline N2-N3 & $2.09(1.05-4.19) ; P=0.037$ & $1.49(0.89-2.48) ; P=0.129$ \\
\hline \multicolumn{3}{|l|}{ HPV status } \\
\hline Positive & 1 & 1 \\
\hline Negative & $2.51(1.38-4.56) ; P=0.003$ & $2.46(1.52-3.98) ; P<0.001$ \\
\hline \multicolumn{3}{|l|}{ Treatment } \\
\hline $\mathrm{Sx} \pm(\mathrm{C}) \mathrm{RT}$ & 1 & 1 \\
\hline (C)RT $\pm S x$ & $1.01(0.56-1.82) ; P=0.98$ & $1.13(0.71-1.81) ; P=0.604$ \\
\hline \multicolumn{3}{|l|}{ TILs } \\
\hline High ( $\geq 20 \%)$ & 1 & 1 \\
\hline Low $(<20 \%)$ & $2.84(1.58-5.11) ; P<0.001$ & $2.27(1.38-3.75) ; P=0.001$ \\
\hline \multicolumn{3}{|c|}{ Multivariable analysis } \\
\hline \multicolumn{3}{|l|}{ T classification } \\
\hline $\mathrm{T} 1$ & 1 & 1 \\
\hline $\mathrm{T} 2$ & $2.19(0.79-6.16) ; P=0.134$ & $1.79(0.72-4.41) ; P=0.208$ \\
\hline T3 & $1.49(0.49-4.43) ; P=0.478$ & $2.01(0.77-5.26) ; P=0.154$ \\
\hline T4 & $2.53(0.87-7.37) ; P=0.089$ & $3.48(1.36-8.87) ; P=0.009$ \\
\hline \multicolumn{3}{|l|}{ HPV status } \\
\hline Positive & 1 & 1 \\
\hline Negative & $2.98(1.58-5.64) ; P=0.001$ & $2.41(1.44-4.05) ; P=0.001$ \\
\hline \multicolumn{3}{|l|}{ TILs } \\
\hline High ( $\geq 20 \%)$ & 1 & 1 \\
\hline Low $(<20 \%)$ & $2.13(1.14-3.96) ; P=0.017$ & $1.87(1.11-3.13) ; P=0.018$ \\
\hline
\end{tabular}

The analyses include overall survival and disease-specific survival for tumour-infiltrating lymphocytes (TILs) and clinicopathologic factors.

allow for a worldwide consensus on the evaluation of TILs in OPSCC. International collaborative efforts are needed to achieve this valuable goal. Of note, such efforts have been undertaken for the assessment of TILs in breast cancer, which has led to an international recommendation [9]. Furthermore, the recent WHO classification of breast tumours recommended the assessment of TILs in daily practice with breast tumours [34]. High reproducibility of results [11-14] and simple technical requirements (just an HEstained slide) as well as an easy and rapid assessment by pathologists make the assessment of TILs a method of great promise. Therefore, future studies on TILs in OPSCC are advised to follow the method introduced by the International ImmunoOncology Working Group.

The immune system is important for the efficacy of cancer therapy [19]. Interestingly, accumulated evidence about the clinical significance of assessment of TILs is of major importance. For example, Denkert et al. reported on the use of TILs in predicting response to treatment in breast cancer [19, 35]. Further, Cha et al. [36] reported that TILs scores in core needle biopsies correspond with the status of TILs in resected breast cancer samples. Similar finding was recently reported by Brcic et al. [37] in OPSCC, and therefore further studies are needed to assess the possibility of using TILs in preoperative biopsies and correlating it with treatment response. Reference images for the assessment of TILs in breast cancer are currently available online (www.tilsinbreastcancer.org) and a similar reference for OPSCC would be welcome. Moreover, a digital image analysis of TILs in $\mathrm{HE}$-slides has been reported in many cancers and a significant correlation with the scores of a human observer [14], but with an even better prognostic value [17]. Such computer-based assessment of TILs should be part of future studies assessing TILs in OPSCC.

Although HPV + OPSCCs have a better survival rate than HPVnegative cases, some cases of HPV + OPSCCs may have an aggressive behaviour [38]. It is of great clinical importance to recognise those HPV + OPSCC cases with good prognosis and therefore eligible to de-escalation therapy (i.e. less intensive treatment with elimination of chemotherapy and/or reduction in radiation [39]). Indeed, successful de-escalation requires an accurate risk-stratification. Findings of the present study indicate that those HPV+ OPSCC patients who could benefit from de-escalation may be identified by assessing their immune response to cancer cells. Our study reports that the TIL score is a good representative of an immune response which significantly relates to patient survival, as also reported elsewhere [40, 41]. We suggest that assessment of TILs should be included in pathology reports and considered in clinical risk stratification of OPSCC. TILs score in OPSCC can be considered for upstaging (in tumours with low TILs) or downstaging (in tumours with high infiltration of TILs). Incorporation of TILs in the TNM classification could be a step towards the introduction of TNM-Immune, as has been considered recently in some cancers [42-44], but not yet in OPSCC. Therefore, future studies with larger multiinstitutional cohorts are necessary.

Indeed, to reach a more precise prognostication it is important to take multiple prognostic factors into consideration including different aspects such as immune-related, cancer-related, and patient-related. TILs score should be considered when deciding the need for adjuvant therapy of OPSCC. In addition, assessment of TILs may also serve as a predictive marker in assessing treatment response in OPSCC cases. Furthermore, evaluation of TILs may be considered in ongoing immunotherapy trials in head and neck cancer [45]. Such prospective clinical trial datasets have high accuracy and reliability for validation of TILs as a biomarker [46]. Interestingly, clinical trials in breast cancer immunotherapy have reported prognostic significance of TILs in HE-stained sections $[46,47]$. Similar evaluations of TILs in trials of head and neck cancer are required.

In conclusion, the assessment of TILs using readily available HE-stained sections is a cost-effective tool that can be used as an immune-based classification for OPSCC. Limitations of our present findings include the retrospective nature of the study, and the fact that it was based on a single-institution cohort. Of note, our findings are supported by recent studies on cancers of the oropharynx $[40,41]$ and other subsites of the head and neck $[12,23,48]$, as well as other locations [14, 19, 32] reporting prognostic usefulness of TILs in HE-stained sections. In addition, the method of assessment used in this study is well-defined and reported to yield good reproducibility and reliability as a prognostic marker in various cancers [11, 14]. Recent research has also confirmed the clinical significance of assessing TILs in OPSCC [49] and other cancers [50, 51]. Therefore, the method used in this study can be considered as a standardised method for further validations in other cohorts of OPSCC to allow future implantation of TILs in daily practice. 


\section{DATA AVAILABILITY}

All data that reported in this study is available from the corresponding author on reasonable request.

\section{REFERENCES}

1. Hong $A$, Lee $C S$, Jones $D$, Veillard AS, Zhang $M$, Zhang $X$, et al. Rising prevalence of human papillomavirus-related oropharyngeal cancer in Australia over the last 2 decades. Head Neck. 2016;38:743-50.

2. Lucas-Roxburgh R, Benschop J, Lockett B, van den Heever U, Williams R, Howe L. The prevalence of human papillomavirus in oropharyngeal cancer in a New Zealand population. PLoS ONE. 2017;12:e0186424.

3. Lu DJ, Luu M, Mita A, Scher K, Shiao SL, Yoshida EP, et al. Human papillomavirusassociated oropharyngeal cancer among patients aged 70 and older: Dramatically increased prevalence and clinical implications. Eur J Cancer. 2018;103:195-204.

4. Faraji F, Rettig EM, Tsai HL, El Asmar M, Fung N, Eisele DW, et al. The prevalence of human papillomavirus in oropharyngeal cancer is increasing regardless of sex or race, and the influence of sex and race on survival is modified by human papillomavirus tumor status. Cancer. 2019;125:761-9.

5. Gillison ML, Chaturvedi AK, Anderson WF, Fakhry C. Epidemiology of human papillomavirus-positive head and neck squamous cell carcinoma. J Clin Oncol. 2015;33:3235-42.

6. Yin LX, D'Souza G, Westra WH, Wang SJ, van Zante A, Zhang Y, et al. Prognostic factors for human papillomavirus-positive and negative oropharyngeal carcinomas. Laryngoscope. 2018;128:E287-E295.

7. Palucka AK, Coussens LM. The basis of oncoimmunology. Cell 2016;164:1233-47.

8. Almangush A, Leivo I, Makitie AA. Overall assessment of tumor-infiltrating lymphocytes in head and neck squamous cell carcinoma: time to take notice. Acta Otolaryngol. 2020;140:246-8.

9. Salgado R, Denkert C, Demaria S, Sirtaine N, Klauschen F, Pruneri G, et al. The evaluation of tumor-infiltrating lymphocytes (TILs) in breast cancer: recommendations by an International TILs Working Group 2014. Ann Oncol. 2015;26:259-71.

10. Hendry S, Salgado R, Gevaert T, Russell PA, John T, Thapa B, et al. Assessing tumorinfiltrating lymphocytes in solid tumors: a practical review for pathologists and proposal for a standardized method from the International Immuno-Oncology Biomarkers Working Group: part 2: TILs in melanoma, gastrointestinal tract carcinomas, non-small cell lung carcinoma and mesothelioma, endometrial and ovarian carcinomas, squamous cell carcinoma of the head and neck, genitourinary carcinomas, and primary brain tumors. Adv Anat Pathol. 2017;24:311-35.

11. Swisher SK, Wu Y, Castaneda CA, Lyons GR, Yang F, Tapia C, et al. Interobserver agreement between pathologists assessing tumor-infiltrating lymphocytes (TILs) in breast cancer using methodology prOPOSED by the International TILs Working Group. Ann Surg Oncol. 2016;23:2242-8.

12. Heikkinen I, Bello IO, Wahab A, Hagstrom J, Haglund C, Coletta RD, et al. Assessment of tumor-infiltrating lymphocytes predicts the behavior of early-stage oral tongue cancer. Am J Surg Pathol. 2019;43:1392-6.

13. Almangush A, Ruuskanen $M$, Hagstrom J, Hirvikoski $P$, Tommola $S$, Kosma VM, et al. Tumor-infiltrating lymphocytes associate with outcome in nonendemic nasopharyngeal carcinoma: a multicenter study. Hum Pathol. 2018;81:211-9.

14. Iseki $Y$, Shibutani M, Maeda K, Nagahara H, Fukuoka T, Matsutani S, et al. A new method for evaluating tumor-infiltrating lymphocytes (TILs) in colorectal cancer using hematoxylin and eosin (H-E)-stained tumor sections. PLoS ONE. 2018;13:e0192744.

15. Gonzalez-Ericsson PI, Stovgaard ES, Sua LF, Reisenbichler E, Kos Z, Carter JM, et al. The path to a better biomarker: application of a risk management framework for the implementation of PD-L1 and TILs as immuno-oncology biomarkers in breast cancer clinical trials and daily practice. J Pathol. 2020;250:667-84.

16. Komforti M, Badve SS, Harmon B, Lo Y, Fineberg S. Tumour-infiltrating lymphocytes in ductal carcinoma in situ (DCIS)-assessment with three different methodologies and correlation with Oncotype DX DCIS Score. Histopathology. 2020;77:749-59.

17. Shaban M, Khurram SA, Fraz MM, Alsubaie N, Masood I, Mushtaq S, et al. A novel digital score for abundance of tumour infiltrating lymphocytes predicts disease free survival in oral squamous cell carcinoma. Sci Rep. 2019;9:13341.

18. Salmon H, Remark R, Gnjatic S, Merad M. Host tissue determinants of tumour immunity. Nat Rev Cancer. 2019;19:215-27.

19. Denkert C, von Minckwitz G, Darb-Esfahani S, Lederer B, Heppner BI, Weber KE, et al. Tumour-infiltrating lymphocytes and prognosis in different subtypes of breast cancer: a pooled analysis of 3771 patients treated with neoadjuvant therapy. Lancet Oncol. 2018;19:40-50.

20. Xu B, Salama AM, Valero C, Yuan A, Khimraj A, Saliba M, et al. Histologic evaluation of host immune microenvironment and its prognostic significance in oral tongue squamous cell carcinoma: a comparative study on lymphocytic host response (LHR) and tumor infiltrating lymphocytes (TILs). Pathol Res Pract. 2021:153473.

21. Fuchs TL, Sioson L, Sheen A, Jafari-Nejad K, Renaud CJ, Andrici J, et al. Assessment of tumor-infiltrating lymphocytes using International TILs Working Group (ITWG) system is a strong predictor of overall survival in colorectal carcinoma: a study of 1034 patients. Am J Surg Pathol. 2020;44:536-44.

22. Miyauchi S, Kim SS, Pang J, Gold KA, Gutkind JS, Califano JA, et al. Immune modulation of head and neck squamous cell carcinoma and the tumor microenvironment by conventional therapeutics. Clin Cancer Res. 2019;25:4211-23.

23. Wang YQ, Chen YP, Zhang Y, Jiang W, Liu N, Yun JP, et al. Prognostic significance of tumor-infiltrating lymphocytes in non-disseminated nasopharyngeal carcinoma: A large-scale cohort study. Int J Cancer. 2018;142:2558-66.

24. Buisseret L, Desmedt C, Garaud S, Fornili M, Wang X, Van den Eyden G, et al. Reliability of tumor-infiltrating lymphocyte and tertiary lymphoid structure assessment in human breast cancer. Mod Pathol. 2017;30:1204-12.

25. Wang J, Tian S, Sun J, Zhang J, Lin L, Hu C. The presence of tumour-infiltrating lymphocytes (TILs) and the ratios between different subsets serve as prognostic factors in advanced hypopharyngeal squamous cell carcinoma. BMC Cancer. 2020;20:731.

26. Peled M, Onn A, Herbst RS. Tumor-infiltrating lymphocytes-location for prog nostic evaluation. Clin Cancer Res. 2019;25:1449-51.

27. Kim JY, Kim CH, Lee Y, Lee JH, Chae YS. Tumour infiltrating lymphocytes are predictors of lymph node metastasis in early gastric cancers. Pathology. 2017:49:589-95.

28. Corredor G, Wang X, Zhou Y, Lu C, Fu P, Syrigos K, et al. Spatial architecture and arrangement of tumor-infiltrating lymphocytes for predicting likelihood of recurrence in early-stage non-small cell lung cancer. Clin Cancer Res. 2019;25:1526-34.

29. Ward MJ, Thirdborough SM, Mellows T, Riley C, Harris S, Suchak K, et al. Tumourinfiltrating lymphocytes predict for outcome in HPV-positive oropharyngeal cancer. Br J Cancer. 2014;110:489-500.

30. Ruangritchankul K, Sandison A, Warburton F, Guerrero-Urbano T, Reis Ferreira M, Lei $M$, et al. Clinical evaluation of tumour-infiltrating lymphocytes as a prognostic factor in patients with human papillomavirus-associated oropharyngeal squamous cell carcinoma. Histopathology. 2019;75:146-50.

31. Faraji F, Fung N, Zaidi M, Gourin CC, Eisele DW, Rooper LM, et al. Tumorinfiltrating lymphocyte quantification stratifies early-stage human papillomavirus oropharynx cancer prognosis. Laryngoscope. 2020;130:930-8.

32. Rakaee M, Kilvaer TK, Dalen SM, Richardsen E, Paulsen EE, Hald SM, et al. Evaluation of tumor-infiltrating lymphocytes using routine H\&E slides predicts patient survival in resected non-small cell lung cancer. Hum Pathol. 2018;79:188-98.

33. Jun SY, Lee EJ, Hong SM, Jung ES, Chung JY. Tumor microenvironmental prog nostic risk in primary operable small intestinal adenocarcinoma. Am J Surg Pathol. 2021:45:917-29.

34. Editorial Board. Breast Tumours (WHO Classification of Tumours). 5th ed. World Health Organization-IARC. 2019;2.

35. Denkert C, von Minckwitz G, Brase JC, Sinn BV, Gade S, Kronenwett R, et al. Tumor-infiltrating lymphocytes and response to neoadjuvant chemotherapy with or without carboplatin in human epidermal growth factor receptor 2-positive and triple-negative primary breast cancers. J Clin Oncol. 2015;33:983-91.

36. Cha YJ, Ahn SG, Bae SJ, Yoon Cl, Seo J, Jung WH, et al. Comparison of tumorinfiltrating lymphocytes of breast cancer in core needle biopsies and resected specimens: a retrospective analysis. Breast Cancer Res Treat. 2018;171:295-302.

37. Brcic I, Gallob M, Schwantzer G, Zrnc T, Weiland T, Thurnher D, et al. Concordance of tumor infiltrating lymphocytes, PD-L1 and p16 expression in small biopsies, resection and lymph node metastases of oropharyngeal squamous cell carcinoma. Oral Oncol. 2020;106:104719.

38. Dagan R, Galloway TJ. Radiation treatment deintensification for HPV-associated oropharyngeal cancer. Semin Radiat Oncol. 2021;31:324-31.

39. Wirth LJ, Burtness B, Nathan CO, Gregoire V, Richmon J. Point/counterpoint: do we de-escalate treatment of HPV-associated oropharynx cancer now? And how? Am Soc Clin Oncol Educ Book. 2019;39:364-72.

40. Kreinbrink PJ, Li J, Parajuli S, Wise-Draper TM, Choi DL, Tang AL, et al. Pretreatment absolute lymphocyte count predicts for improved survival in human papillomavirus (HPV)-driven oropharyngeal squamous cell carcinoma. Oral Oncol. 2021;116:105245

41. De Keukeleire SJ, Vermassen T, De Meulenaere A, Deron P, Huvenne W, Duprez F, et al. Tumour infiltrating lymphocytes in oropharyngeal carcinoma: prognostic value and evaluation of a standardised method. Pathology. 2021;53:836-43.

42. Taube JM. Emerging immunologic biomarkers: setting the (TNM-immune) stage. Clin Cancer Res. 2014;20:2023-5.

43. Galon J, Mlecnik B, Bindea G, Angell HK, Berger A, Lagorce C, et al. Towards the introduction of the 'Immunoscore' in the classification of malignant tumours. J Pathol. 2014;232:199-209.

44. Donnem T, Kilvaer TK, Andersen S, Richardsen E, Paulsen EE, Hald SM, et al. Strategies for clinical implementation of TNM-Immunoscore in resected nonsmall-cell lung cancer. Ann Oncol. 2016;27:225-32.

45. Lee NY, Ferris RL, Psyrri A, Haddad RI, Tahara M, Bourhis J, et al. Avelumab plus standard-of-care chemoradiotherapy versus chemoradiotherapy alone in patients 
with locally advanced squamous cell carcinoma of the head and neck: a randomised, double-blind, placebo-controlled, multicentre, phase 3 trial. Lancet Oncol. 2021;22:450-62.

46. Luen SJ, Salgado R, Fox S, Savas P, Eng-Wong J, Clark E, et al. Tumour-infiltrating lymphocytes in advanced HER2-positive breast cancer treated with pertuzumab or placebo in addition to trastuzumab and docetaxel: a retrospective analysis of the CLEOPATRA study. Lancet Oncol. 2017;18:52-62.

47. Adams S, Gray RJ, Demaria S, Goldstein L, Perez EA, Shulman LN, et al. Prognostic value of tumor-infiltrating lymphocytes in triple-negative breast cancers from two phase III randomized adjuvant breast cancer trials: ECOG 2197 and ECOG 1199. J Clin Oncol. 2014;32:2959-66.

48. Vassilakopoulou M, Avgeris M, Velcheti V, Kotoula V, Rampias T, Chatzopoulos K, et al. Evaluation of PD-L1 expression and associated tumorinfiltrating lymphocytes in laryngeal squamous cell carcinoma. Clin Cancer Res. 2016;22:704-13.

49. Yin LX, Rivera M, Garcia JJ, Bartemes K, Lewis DB, Lohse CM, et al. The impact of tumor infiltrating lymphocytes (TILs) on disease progression in human papillomavirus (HPV)-related oropharyngeal squamous cell carcinoma. J Clin Oncol. 2021;39:6049-6049.

50. Azarianpour-Esfahani S, Fu P, Mahdi H, Madabhushi A. Computational features of TIL architecture are differentially prognostic of uterine cancer between African and Caucasian American women. J Clin Oncol. 2021;39:5585-5585.

51. Sinicrope FA, Shi Q, Smyrk TC, Goldberg RM, Cohen SJ, Gill S, et al. Association of plasma adiponectin with tumor infiltrating lymphocytes and survival in patients with stage III colon cancer (NCCTG N0147; Alliance). J Clin Oncol. 2021;39:3591-3591.

\section{ACKNOWLEDGEMENTS}

We thank the funders of this study (listed below). This study was funded by Turku University Hospital Fund, Helsinki University Hospital Research Fund, the Sigrid Juselius Foundation, the Finnish Cancer Society, Finska Läkaresällskapet, K. Albin Johansson Foundation, and the Maritza and Reino Salonen Foundation.

\section{AUTHOR CONTRIBUTIONS}

Conceptualisation and study design: $\mathrm{AA}, \mathrm{AM}, \mathrm{JH}, \mathrm{CH}$ and IL. Data production, analysis and interpretation: $\mathrm{L}, \mathrm{TA}, \mathrm{AA}, \mathrm{AM}$ and IL. Manuscript writing: $\mathrm{AA}, \mathrm{L}, \mathrm{TA}, \mathrm{AM}$ and IL. Reviewing and editing of the final manuscript: $\mathrm{AM}, \mathrm{CH}, \mathrm{TA}, \mathrm{JH}$ and IL. All authors have reviewed the manuscript and approved the final manuscript.

\section{FUNDING INFORMATION}

This study was funded by Turku University Hospital Fund, Helsinki University Hospital Research Fund, the Sigrid Juselius Foundation, the Finnish Cancer Society, Finska Läkaresällskapet, K. Albin Johansson Foundation, and the Maritza and Reino Salonen
Foundation. Open Access funding provided by University of Helsinki including Helsinki University Central Hospital.

\section{ETHICS APPROVAL AND CONSENT TO PARTICIPATE}

Ethical approval for this study was obtained from the Research Ethics Committee of the Helsinki University Hospital. The study was performed in accordance with the Declaration of Helsinki. Patient consent was not required, and this is approved by the ethics committee because researchers working on anonymised data in this retrospective research.

\section{CONSENT TO PUBLISH}

All authors confirm that this manuscript has not been published anywhere else, and it is not being considered for publication elsewhere. All persons listed as authors have approved the manuscript and agree with its publication in this journal.

\section{COMPETING INTERESTS}

The authors declare no competing interests.

\section{ADDITIONAL INFORMATION}

Correspondence and requests for materials should be addressed to Alhadi Almangush.

Reprints and permission information is available at http://www.nature.com/ reprints

Publisher's note Springer Nature remains neutral with regard to jurisdictional claims in published maps and institutional affiliations.

\section{()}

Open Access This article is licensed under a Creative Commons Attribution 4.0 International License, which permits use, sharing, adaptation, distribution and reproduction in any medium or format, as long as you give appropriate credit to the original author(s) and the source, provide a link to the Creative Commons license, and indicate if changes were made. The images or other third party material in this article are included in the article's Creative Commons license, unless indicated otherwise in a credit line to the material. If material is not included in the article's Creative Commons license and your intended use is not permitted by statutory regulation or exceeds the permitted use, you will need to obtain permission directly from the copyright holder. To view a copy of this license, visit http://creativecommons. org/licenses/by/4.0/.

(c) The Author(s) 2022 